\title{
Transição da morbimortalidade no Brasil: um desafio aos 30 anos de SUS
}

\author{
Transition of morbidity and mortality in Brazil: \\ a challenge on the thirtieth anniversary of the SUS
}

Thalyta Cássia de Freitas Martins (https://orcid.org/0000-0002-6225-7245) ${ }^{1}$

José Henrique Costa Monteiro da Silva (https://orcid.org/0000-0001-9674-3041) ${ }^{2}$

Geovane da Conceição Máximo (https://orcid.org/0000-0002-4330-9927) ${ }^{3}$

Raphael Mendonça Guimarães (https://orcid.org/0000-0003-1225-6719) ${ }^{4}$

${ }^{1}$ Programa de PósGraduação em Saúde Pública, Escola Nacional de Saúde Pública Sergio Arouca, Fundação Oswaldo Cruz. R. Leopoldo Bulhões 1.480, Manguinhos. 21041210 Rio de Janeiro RJ Brasil.raphael.guimaraes@ fiocruz.br

${ }^{2}$ Centro Latino-Americano e Caribe para Demografia. Santiago Chile.

${ }^{3}$ Departamento de Geografia, Universidade Federal dos Vales do Jequitinhonha e Mucuri. Alto da Jacuba Diamantina MG Brasil.

${ }^{4}$ Escola Politécnica de Saúde Joaquim Venâncio, Fundação Oswaldo Cruz. Rio de Janeiro RJ Brasil.

\begin{abstract}
In 2020, the 30th anniversary of the publication of the Organic Laws of the Unified Health System was celebrated. Since then, the change in the profile of morbidity and mortality has been a challenge to management to ensure that the health services can attend the significant heterogeneity of approximately 6,000 municipalities. To achieve this, it is necessary to monitor the leading indicators of the country. The scope of this study was to present an overview of trends in mortality and morbidity in Brazil between 1990 and 2019. Data from the Study on the Global Burden of Disease was used to describe morbidity and mortality by major groupings (infectious diseases, chronic diseases, and external causes), according to gender and age groups. There was a reduction in morbidity and mortality in the period, irrespective of the cause or age group, albeit with a varied difference between the sexes depending on the cause. The contribution of chronic diseases increases with age, with a marked difference according to gender. The curves for mortality and years lost due to disability have a typical profile, with a different pattern of curves for men due to external causes, with marked excess mortality at young ages. The trend confirms the decline of indicators in a linear manner over the period.
\end{abstract}

Key words Health transition, Cause of death, Demographic analysis, DALY, Mortality
Resumo Em 2020, completamos 30 anos desde a publicação das Leis Orgânicas do Sistema Único de Saúde. Desde então, a mudança no perfil de morbimortalidade tem desafiado a gestão, para que o serviço de saúde consiga atender à grande heterogeneidade dos quase seis mil municipios. Para isso, é necessário monitorar os principais indicadores do país. O objetivo do presente estudo foi apresentar uma visão geral das tendências de mortalidade e morbidade no Brasil entre 1990 e 2019. Utilizamos os dados do Estudo de Carga Global de Doenças para descrever a morbimortalidade pelos grandes grupos (doenças infecciosas, doenças crônicas e causas externas), segundo sexo e grupos etários. Há redução da morbimortalidade no período, independente do grupo de causa ou faixa etária, com variada diferença entre sexo de acordo com o grupo de causas. A contribuição das doenças crônicas é crescente com a progressão da idade, com diferença substancial segundo o sexo. As curvas de mortalidade e de anos perdidos por incapacidade possuem padrão típico, com destaque ao padrão diferenciado para curvas de homens por causas externas, com marcada sobremortalidade em idades jovens. A tendência ratifica o declinio dos indicadores de forma linear no período.

Palavras-chave Transição da saúde, Causas de morte, Análise demográfica, DALY, Mortalidade 


\section{Introdução}

A transição demográfica e o envelhecimento populacional são evidentes em todo o mundo. Nas últimas seis décadas, a expectativa de vida da população mundial aumentou de 47 anos, no período 1950-1955, para cerca de 72 anos, entre 2015-2020. Ainda assim, esse aumento se deu de forma distinta entre países e revela heterogeneidades marcantes de nível. Por exemplo, enquanto os países de renda alta atingiram níveis de expectativa de vida ao nascer superiores a 80 anos em 2015-2020, os países de renda média apresentaram valores em torno de 71 anos e os países de renda baixa valores em torno de 63 anos $^{1}$. Essas disparidades refletem as distintas trajetórias demográficas e epidemiológicas dos países.

Os aumentos na longevidade foram acompanhados por mudanças nos padrões e riscos de mortalidade por causas específicas nas populações humanas, documentadas e descritas por marcos teóricos que complementam a teoria da transição demográfica. Destacam-se a transição epidemiológica $^{2}$ e a transição da saúde ${ }^{3}$. Essas teorias foram amplamente utilizadas por pesquisadores de diferentes perspectivas disciplinares para estudar e comparar as experiências de saúde e população de vários países, e vêm sendo utilizadas na abordagem de estudos de mortalidade, morbidade, saúde e desenvolvimento, desenvolvimento populacional e desenvolvimento da saúde nos contextos de países desenvolvidos, em países de renda média em rápida mudança e, em menor grau, em países de baixa renda ${ }^{4}$.

O conceito de transição epidemiológica foi utilizado a partir da década de 1970 para descrever e explicar as transformações nos padrões de ocorrência de doenças e causas de morte ${ }^{2,5,6}$. De modo geral, trata-se de uma estrutura conceitual para discutir como os padrões de doenças se modificaram ao longo do tempo, passando de um padrão marcado por enfermidades predominantemente infecciosas para um padrão de maior prevalência de doenças crônicas não transmissíveis.

A teoria de transição epidemiológica vem sendo revisitada com propostas de extensões que levem em consideração os aspectos mais recentes observados nos perfis de envelhecimento das populações, como aumento da expectativa de vida saudável (livre de morbidades) ${ }^{3,7-9}$. Contudo, desde a década de 1980, essa teoria tem sua aplicabilidade contestada em países de baixa e média renda (LMICs), onde o monitoramento da mortalidade e da morbidade da população é inexistente ou produz dados incompletos ou de baixa confiabilidade. Além disso, questiona-se a validade do modelo proposto por $\mathrm{Omran}^{2}$ por este não levar em conta a importância das crenças e valores culturais e sociais, das forças políticas socioeconômicas e das políticas de saúde na compreensão dos distintos perfis epidemiológicos, especialmente nos países em desenvolvimento ${ }^{10}$. Por outro lado, os contextos sociais, econômicos, políticos, culturais e demográficos não podem ser deixados de lado nas discussões dos padrões de saúde, doença e mortalidade naqueles países e levam a reflexões distintas da retórica construída com base em informações de nações de alta renda.

Esses aspectos são marcas dos múltiplos cenários de transições epidemiológica, demográfica e de saúde que também se constatam no Brasil. Apesar de experimentar uma transição de um padrão de mortalidade e morbidade por enfermidades infecciosas para um perfil com maior relevância de doenças crônicas e não transmissíveis, no geral, o país convive com distintos padrões de transição que são o resultado de diferenças de nível de desenvolvimento regional e social ${ }^{11}$.

O Sistema Único de Saúde (SUS) brasileiro obteve inúmeras conquistas ao longo das suas três décadas. Desde a publicação das Leis Orgânicas de Saúde, em 1990, houve um aumento progressivo na cobertura de serviços de saúde para a população, de forma universal e em todos os níveis de complexidade ${ }^{12}$. Contudo, persistem desafios até os dias atuais. Um deles, em particular, chama a atenção em função de ser um balizador importante para se traçar metas de planejamento de ações assistenciais e de vigilância em saúde: a transição epidemiológica e, mais ainda, a transição da saúde. Isto significa dizer que não apenas o padrão de mortalidade, mas também o de morbidade, vem se modificando de forma notória nos últimos anos no Brasil ${ }^{13}$. Com base no arcabouço teórico apresentado, o presente estudo se propõe a apresentar uma visão geral das tendências de mortalidade e morbidade no Brasil no período de 1990 a 2019.

\section{Metodologia}

\section{Fonte de dados}

O estudo utilizou as estimativas do estudo Carga Global de Doença (GBD) para o Brasil e estados, para um período de 30 anos (1990 a 2019). Este estudo analisa um conjunto de cerca 
de 250 causas de morte, mais de 70 fatores de risco em mais de 190 países $^{14}$. Vale ressaltar que este período coincide com a publicação das Leis Orgânicas de Saúde, de forma que a evolução temporal descreve o período da história do próprio sistema de saúde brasileiro.

Foram utilizadas duas métricas disponibilizadas pelo estudo de Carga Global de Doenças. Primeiramente, realizou-se a extração das taxas de mortalidade, por faixa etária quinquenal, e a respectiva padronização, pelo método direto, utilizando a população brasileira do meio do período analisado como população padrão, ou seja, a população estimada em 2004. Em seguida, repetiu-se a mesma operação para o indicador de anos de vida ajustados por incapacidade (DALY). Trata-se de um indicador que estima a carga de doença, pois reúne em si o peso da mortalidade prematura e, também, do número de anos que as pessoas vivem com incapacidades decorrentes das doenças que elas tem, levando a um estado de saúde que não é pleno ${ }^{15}$. Para o estudo adequado da mortalidade, a faixa etária de 0 a 4 anos foi dividida entre menores de 1 ano e entre 1 a 4 anos, para que fosse possível destacar a mortalidade infantil. Além disso, a última faixa etária se trata de intervalo aberto para pessoas com 80 anos ou mais de idade.

\section{Análise dos dados}

Dividimos as causas em três grandes grupos correspondentes ao nível 1 do GBD: doenças infecciosas, doenças crônicas não transmissíveis e causas externas. Extraímos os dados por faixa etária quinquenal e por sexo, para todos os anos do período entre 1990 e 2019. As análises foram realizadas estratificando-se por sexo e faixa etária. Numa primeira etapa, realizamos as análises para os anos de 1990 e 2019. Inicialmente, realizamos o cálculo da taxa para o DALY e a mortalidade, ajustada para idade. Tomamos como população padrão a população mundial apresentada por Doll et al. ${ }^{16}$. Realizamos então uma inspeção visual da série temporal.

A fim de descrever a diferença nos anos inicial e final da série, calculamos a variação entre estes dois anos, para cada grupo de causas, sexo e faixa etária. Com isso pudemos verificar quais grupos de causas sofreram maior variação no ano de início do SUS e no seu $30^{\circ}$ aniversário, caracterizando a transição da morbimortalidade. Para corroborar esta análise, estimamos a mortalidade proporcional e a proporção de DALY por faixa etária e sexo para os anos de 1990 e 2019.
Para avaliar o comportamento dos grupos de causas, estimamos curvas da mortalidade e dos anos de incapacidade (DALY), estimamos curvas logarítmicas que descrevessem o padrão para cada grupo de causas. A transformação logarítmica foi realizada de forma a possibilitar a análise comparativa das faixas etárias. Uma vez que a ordem de grandeza da mortalidade de crianças, adolescentes e adultos jovens é substancialmente menor que das idades mais avançadas, este procedimento é importante para tornar possível a visualização das taxas na apresentação gráfica.

Em uma última etapa, procuramos observar a variação destes indicadores no tempo. Desta forma, realizamos uma análise de séries temporais ${ }^{17}$. Verificamos o padrão da série histórica, para garantir que não se tratava de séries estacionárias. Para isso, foi aplicado o teste de Wald-Wolfowitz. Em seguida, com o objetivo de se obter uma primeira ideia da evolução dos valores das taxas das séries, avaliando o efeito da tendência, foi realizado o teste de Cox-Stuart.

Para a análise das tendências, cuja variável dependente $(Y)$ é representada pelas taxas de mortalidade e a taxa de DALY, e a variável independente $(\mathrm{X})$ é representada pelos anos-calendário, foram construídos diagramas de dispersão entre as taxas e os anos de estudo. Ao verificar a direção da tendência, pudemos constatar que as séries são lineares.

A fim de evitar a autocorrelação serial, optamos por usar a regressão linear generalizada de Prais-Winsten, em substituição à regressão linear simples. Avaliamos a autocorrelação por meio da estatística de Durbin-Watson, obtida no resultado da regressão de Prais-Winsten. As taxas de incremento anual das taxas, bem como os respectivos intervalos de confiança foram obtidas aplicando-se, as fórmulas:

$$
\begin{aligned}
& \text { Taxa de Incremento Anual }=-1+10^{\beta} \\
& \mathrm{IC}_{95 \%}=-1+10^{(\beta \pm \mathrm{t}(0,05 ; \mathrm{n}-1) \mathrm{x} \text { EP })}
\end{aligned}
$$

O coeficiente de regressão (beta) e o erro padrão (EP) da estimativa beta foram fornecidos pela regressão de Prais-Winsten, e o valor de $t$ foi obtido através da tabela da distribuição $t$ de Student bicaudal, com 5\% de nível de significância, considerando-se o número de anos da série menos um.

Utilizamos o software para a análise dos dados o $R^{16}$, versão 3.5 .3 , com manipulação de dados no Excel. 


\section{Resultados}

Entre 1990 e 2019, o Brasil passou por uma série de mudanças e avanços na estruturação de seu sistema de saúde pública e de proteção social básica, que culminaram no incremento dos indicadores globais de mortalidade observados. A Figura 1 apresenta essa evolução em termos das taxas ajustadas de mortalidade e anos de vida perdidos ajustados por incapacidade (do inglês Disability-Adjusted Life Year, DALY), totais e por causa, estratificadas por sexo, entre 1990-2019. Os diferenciais por sexo mostram a vantagem feminina, tanto para o cenário de mortalidade, quanto para a DALY, total e por causas. Notadamente, o maior diferencial por sexo observado está nas causas externas, em que os patamares de mortalidade e DALY dos homens apresentam magnitudes de 3,5 a 4,5 vezes maiores em todo o período.

No que diz respeito à evolução da composição das causas de morte com relação ao total observado, nota-se que para ambos os sexos o DALY das doenças crônicas e não transmissíveis representava aproximadamente 58\% do DALY total em 1990, passando para $69 \%$ em 2019. Nesse mesmo período, a mortalidade por esse mesmo grupo de causas representou $71 \%$ das mortes de 1990 e 75\% em 2019. O DALY de causas externas, por outro lado, oscilou em menor nível no período, passando de $13 \%$ para $15 \%$ do total, tal qual a mortalidade pelas mesmas causas, que passou de $10 \%$ para $12 \%$ do total para ambos os

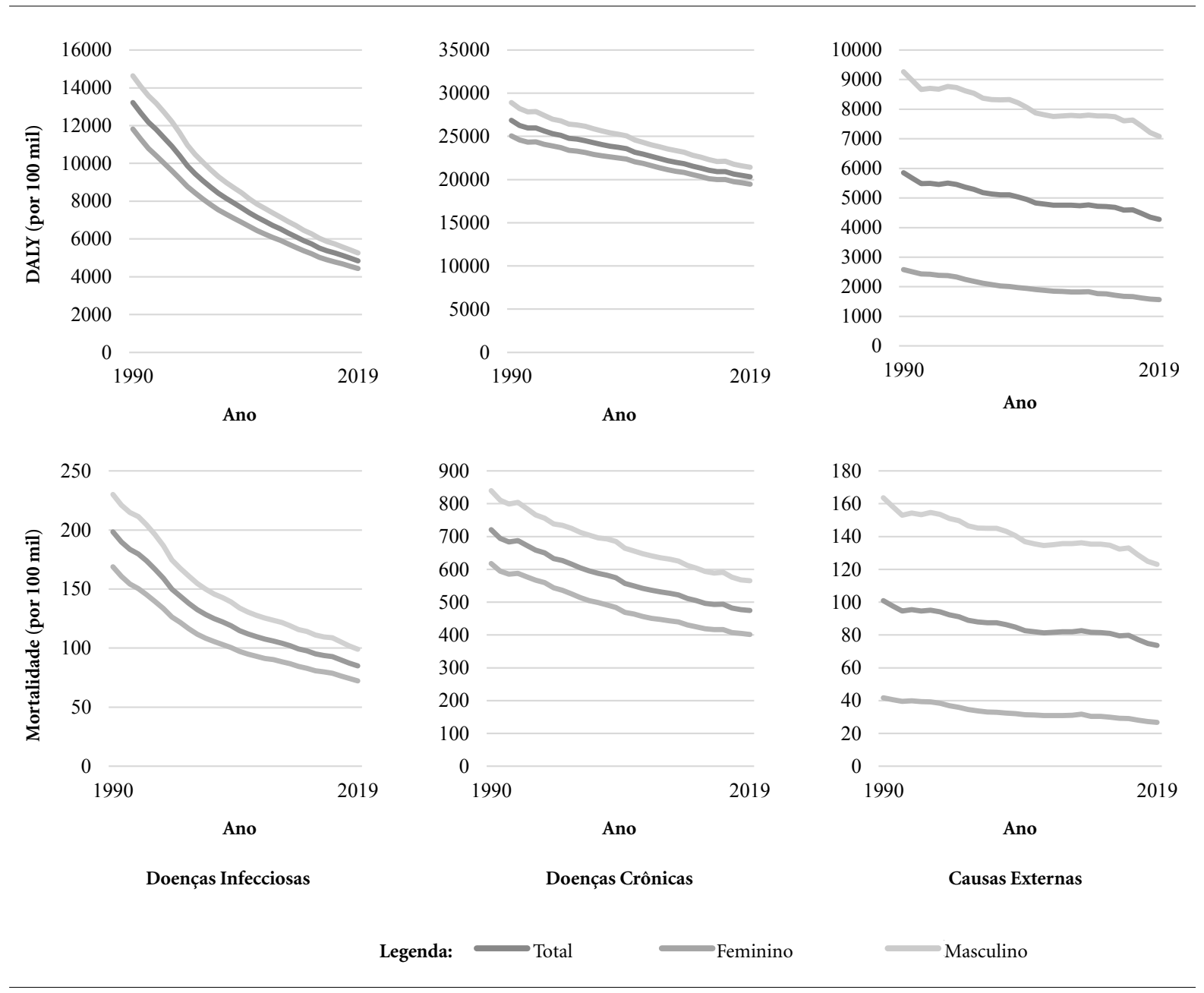

Figura 1. Evolução histórica das taxas de mortalidade e DALY segundo sexo e grupo de causa. Brasil, 1990-2019.

Fonte: GBD, 2021 
sexos. Ademais, o DALY de doenças infecciosas e parasitárias apresentou queda de $29 \%$ do total para $16 \%$ e, a sua mortalidade, passou de $19 \%$ para $13 \%$.

Tanto para o cenário de aumento observado para composição da mortalidade e DALY por doenças crônicas e não transmissíveis, quanto para o cenário de redução para a composição dos indicadores para doenças infecciosas e parasitárias, destaca-se o aumento e a queda mais acentuados para o DALY do que para a mortalidade. Esse comportamento se constitui em evidência do aumento da carga de doenças crônicas e não transmissíveis na morbidade da população do país, concomitante à queda da carga de doenças infecciosas. Ou seja, observa-se um maior peso dos efeitos da morbidade sobre a construção das estimativas de DALY do que da mortalidade em si.

Analisando a variação por idades das mudanças observadas entre 1990 e 2019 (Figura 2), percebe-se um cenário geral de redução nos patamares de DALY e mortalidade ajustada por causas, entre 1990 e 2019. Destaca-se, no entanto, uma estabilidade ou, em alguns casos, um pequeno aumento desses indicadores para os jovens, do sexo masculino, dos grupos etários de 15 a 24 anos. De forma geral, as maiores quedas se deram nos grupos etários de crianças e jovens menores de 15 anos de idade, sobretudo por causas externas, e às doenças infecciosas, enquanto as menores reduções ocorreram nos grupos de idades mais avançadas. Com relação à composição da mortalidade e DALY por grupos de causa (valores proporcionais, Figura 3), destacam-se, sobretudo, os efeitos das infecciosas na mortalidade infantil e de menores de 10 anos, das causas externas na população masculina de jovens adultos e, das doenças crônicas entre a população idosa, em geral, além dos diferenciais por sexo.

A carga de doenças infecciosas e parasitárias, tanto no DALY quanto na mortalidade ajustada, se destacam nas faixas etárias de crianças menores que 10 anos e, principalmente, entre as menores de 1 ano. Para ambos os indicadores, essas causas representavam cerca de $80 \%$ da mortalidade e do valor total do DALY para menores de 1 ano em 1990, reduzindo sua participação para cerca de 66\% em 2019, ao passo que aumentou a contribuição relativa de doenças crônicas e não transmissíveis de $20 \%$ para $30 \%$, no mesmo período. Em geral, as doenças infecciosas e parasitárias reduziram sua contribuição relativa tanto na mortalidade quanto no DALY.

Nos grupos etários entre 15 e 50 anos, nota-se um pequeno aumento/estabilidade da contribui- ção relativa das causas externas para a população masculina. Em 1990, o indicador DALY por causas externas representava 55\% e 59\% do DALY total para os grupos etários de homens entre 15-19 e 20-24, respectivamente, e, em 2019, tais valores representaram 57\% e $60 \%$ do DALY total. Ainda, a mortalidade por causas externas, em 1990, para os mesmos grupos, representou 79\% do total da mortalidade para os homens, passando para cerca de $84 \%$ em 2019. Para as idades mais avançadas, o cenário foi de estabilidade para todos os grupos de causa nos dois indicadores observados. Nota-se que a partir dos 40 anos a contribuição das doenças crônicas passa a ser mais destacada e aumenta conforme a idade.

Na Figura 4 são apresentadas as curvas das taxas específicas de mortalidade por sexo e grupos de causa, para 1990 e 2019. Para a mortalidade total, verificou-se a queda da mortalidade em todos os grupos etários, à exceção das faixas etárias de 15 a 29 anos, que se mantiveram estáveis ou com quedas menos acentuadas. Esse efeito é reflexo do que se verifica para as curvas por causas externas, notadamente, para a população masculina. A despeito da queda observada para as faixas etárias em menores de 15 anos, a mortalidade masculina por causas externas manteve-se em patamares semelhantes, tanto em 1990, quanto em 2019, com pequenas quedas para os grupos etários de maiores de 30 anos. Por outro lado, as doenças infecciosas exibiram quedas em suas taxas para todos os grupos etários entre 1990 e 2019, principalmente entre as crianças e jovens. Para os idosos, essa redução foi menor. Por fim, as taxas de mortalidade específicas por doenças crônicas e não transmissíveis também apresentaram queda no período, mas em uma escala mais reduzida do que se observou para as doenças infecciosas e parasitárias.

De modo geral, o que se observou no período de 1990 a 2019 foi um encolhimento da contribuição das doenças infecciosas e parasitárias para os níveis de mortalidade e DALY observados. Para as crianças e jovens, essa contribuição foi absorvida pelo aumento das contribuições por doenças crônicas e não transmissíveis, que observaram uma redução de suas taxas em um menor grau. Nas faixas etárias de jovens e adultos homens, a estabilidade das taxas de mortalidade por causas externas fez com que esse grupo de causa mantivesse ou aumentasse a sua participação relativa com relação aos totais de mortalidade e DALY observados para os respectivos grupos etários. Já nas idades mais avançadas, o que se observa é um cenário de estabilidade na contri- 


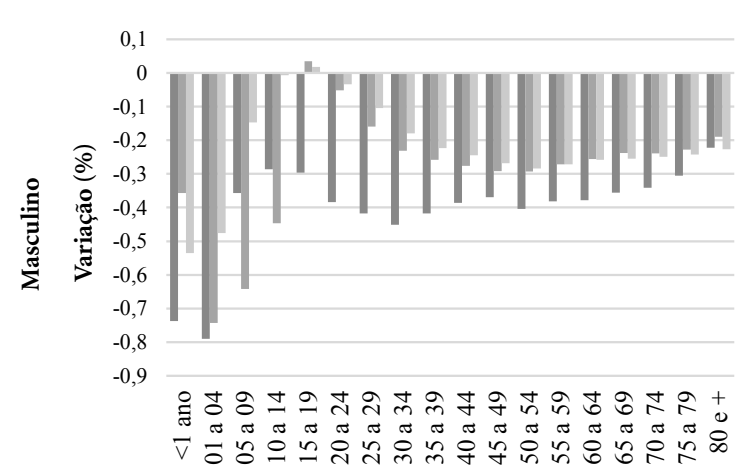

Grupo Etário

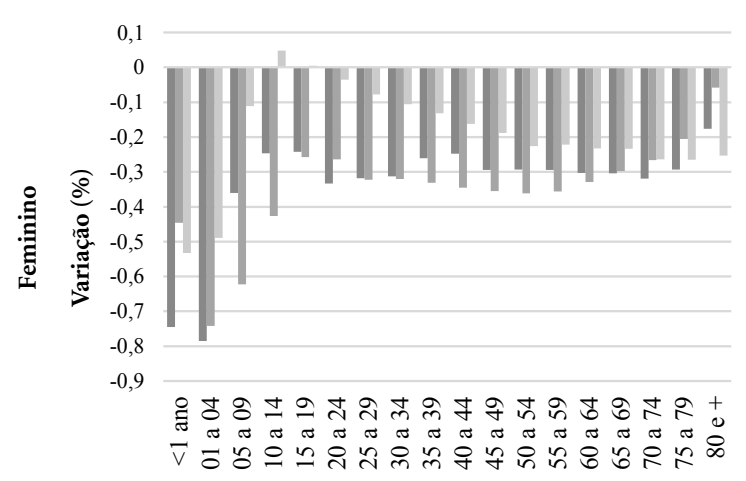

Grupo Etário

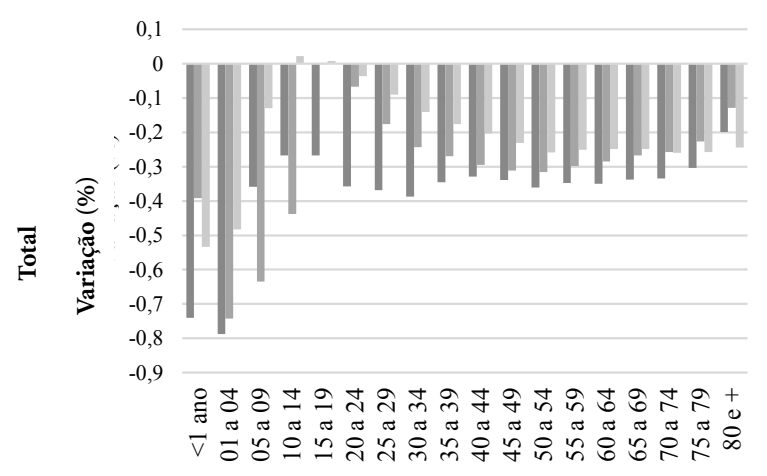

Grupo Etário

DALY

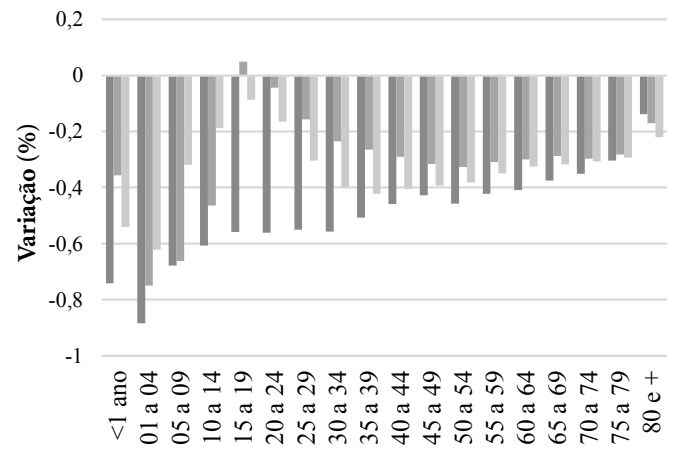

Grupo Etário

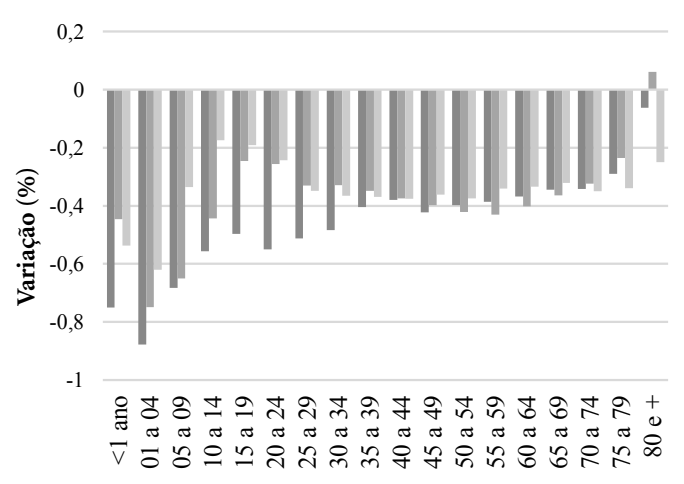

Grupo Etário

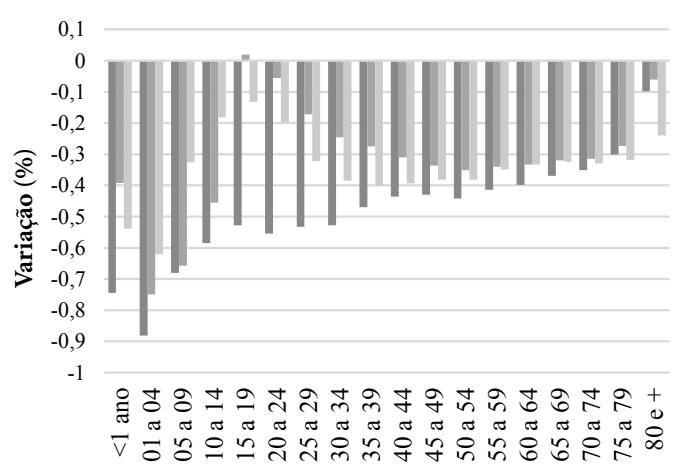

Grupo Etário

Mortalidade

Legenda: 2 Infecciosas DCNT 2 Externas

Figura 2. Variação das taxas de mortalidade e DALY segundo grupos de causa por sexo e grupos etários. Brasil, 1990-2019.

Fonte: GBD, 2021.

buição relativa de cada grupo de causa, com quedas pequenas nas taxas de mortalidade e DALY no período analisado.

Finalmente, para todas as categorias de sexo e grupo de causas, a tendência das taxas de morta- lidade e o DALY ajustado por idade apresentam tendência de queda (Tabela 1). Todos apresentaram queda estatisticamente significativa, com ótimo ajuste do modelo linear. Com relação ao sexo, as variações em mulheres são maiores que entre 

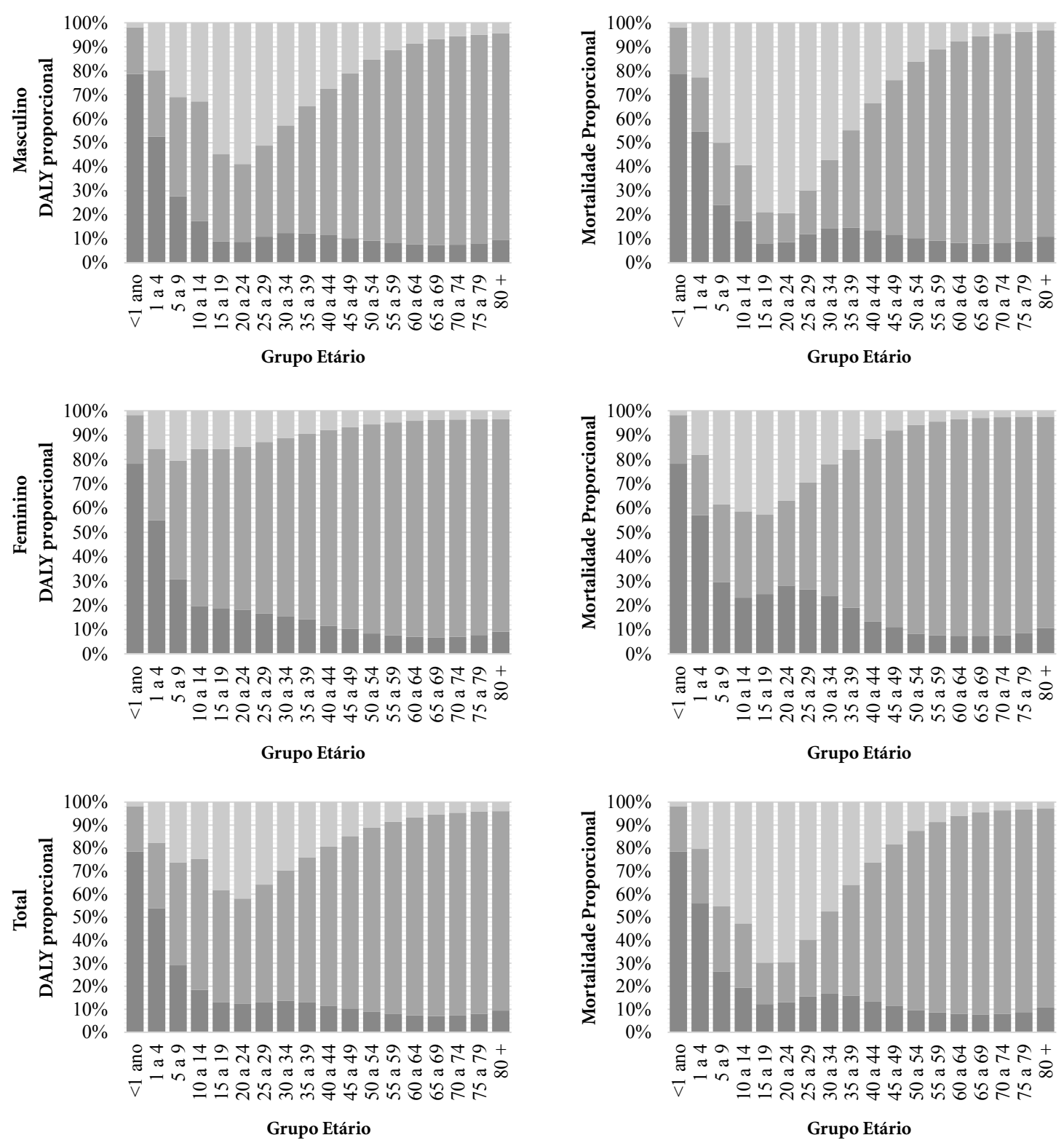

DALY

Mortalidade

1990

Figura 3. Mortalidade proporcional e DALY por grupos de causa segundo sexo e grupos etários. Brasil, 1990-2019.

homens para doenças infecciosas. Para os demais grupos de causas, a variação dos homens possuiu maior variação. O grupo das causas externas é aquele que apresenta redução mais consistente dentre os grupos de causa. Destacamos a variação média anual de $3,41 \%$ (IC 95\% -3,51; -3,32) para o DALY, como sendo aquele grupo com maior variação de queda. A menor variação de queda foi a mortalidade para doenças infecciosas e parasitárias, de $-0,86 \%$ (IC 95\% -0,88; -0,84).

\section{Discussão}

Observou-se que entre os anos de 1990 e 2019 o aumento do DALY de doenças crônicas não 


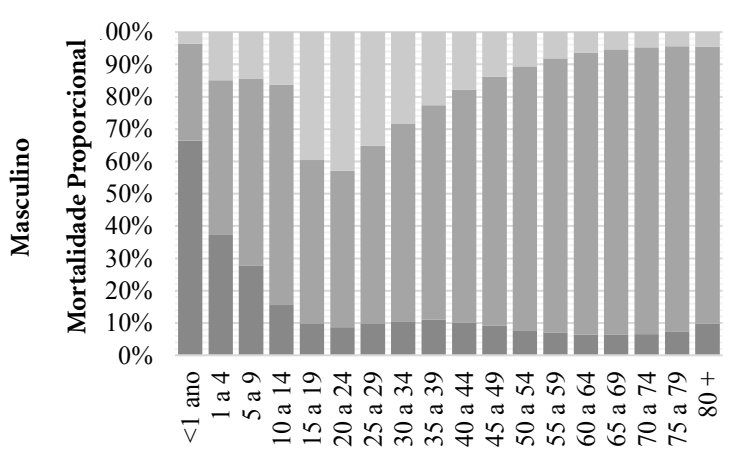

Grupo Etário

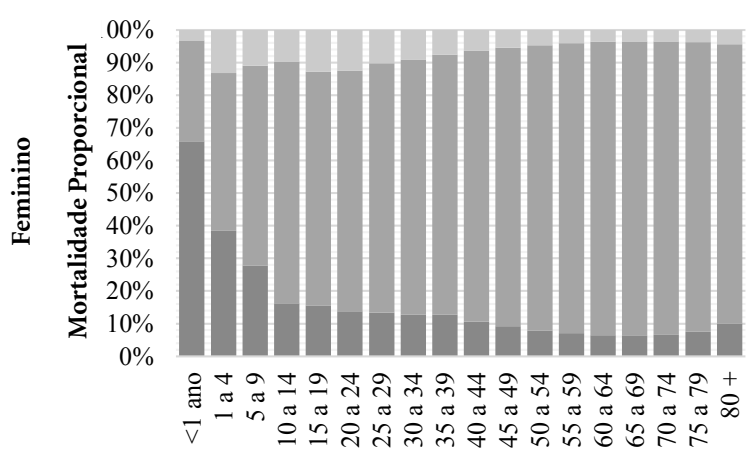

Grupo Etário

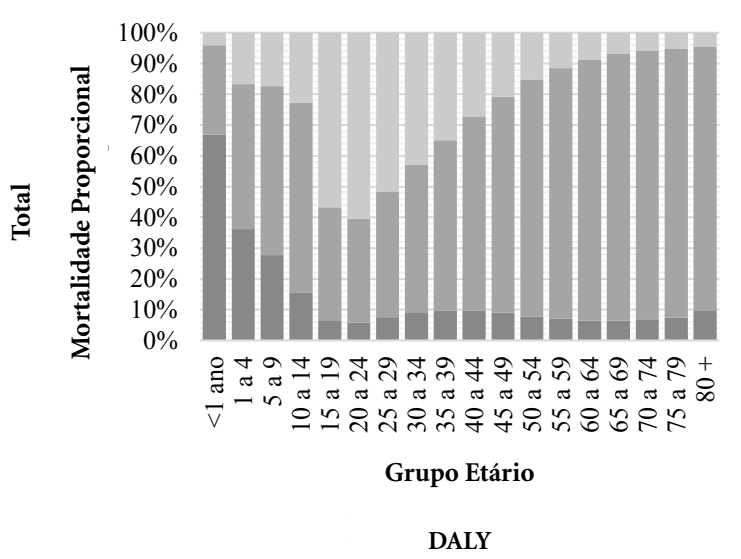

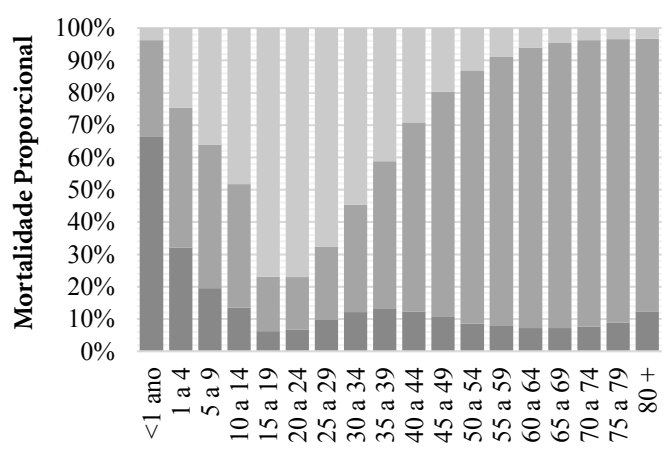

Grupo Etário

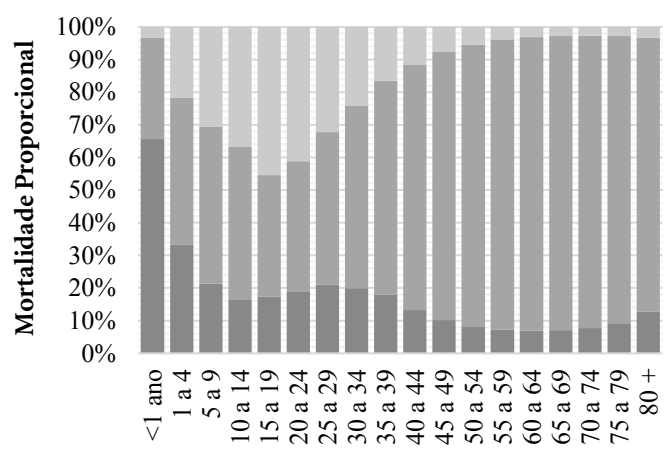

Grupo Etário

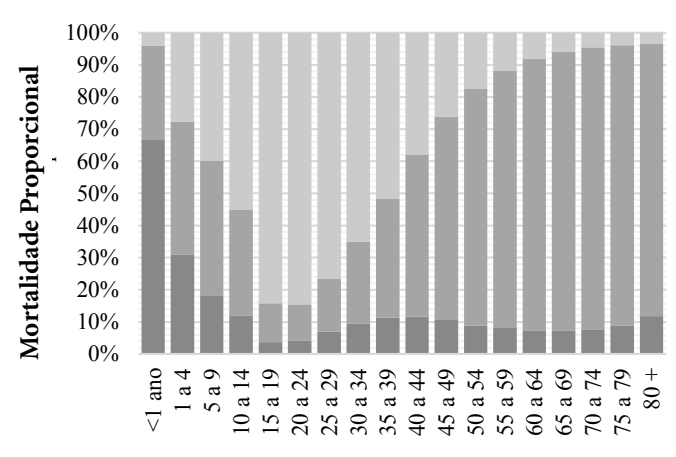

Grupo Etário

Mortalidade

2019

Legenda: Infecciosas $\longrightarrow$ DCNT $\longrightarrow$ Externas

Figura 3. Mortalidade proporcional e DALY por grupos de causa segundo sexo e grupos etários. Brasil, 1990-2019.

Fonte: GBD, 2021.

transmissíveis e causas externas foi proporcionalmente maior quando comparado ao aumento da mortalidade nesses mesmos grupos de causas, indicando um aumento da carga de doenças crônicas não transmissíveis na morbidade da população do país. O diagnóstico de mudanças no padrão de morbimortalidade brasileiro não é recente. O estudo de mudança do perfil da carga de doenças no Brasil entre os anos de 1990 a 2010, em que observou-se que os brasileiros passaram a viver mais tempo e, em média, a morrer com idade mais avançada ${ }^{18}$. No entanto, a relação en- 


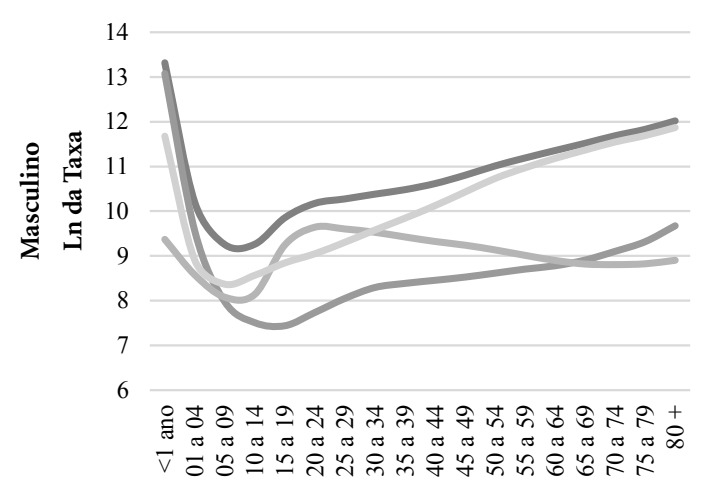

Grupo Etário

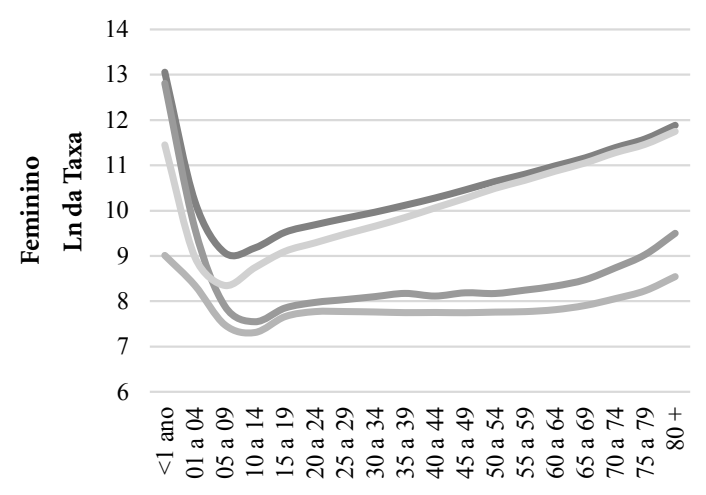

Grupo Etário

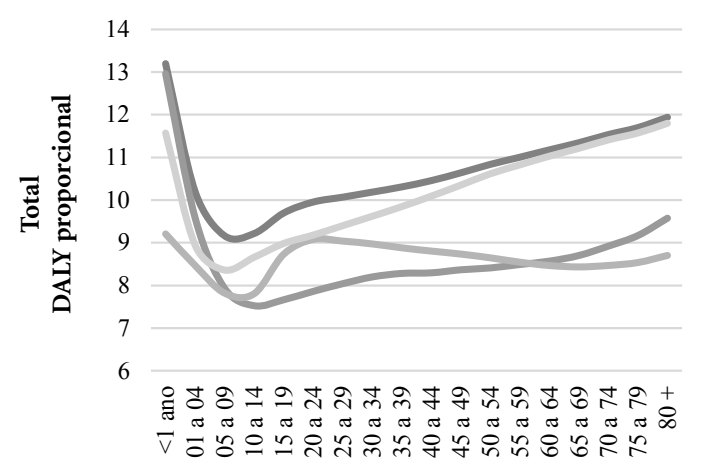

Grupo Etário

DALY

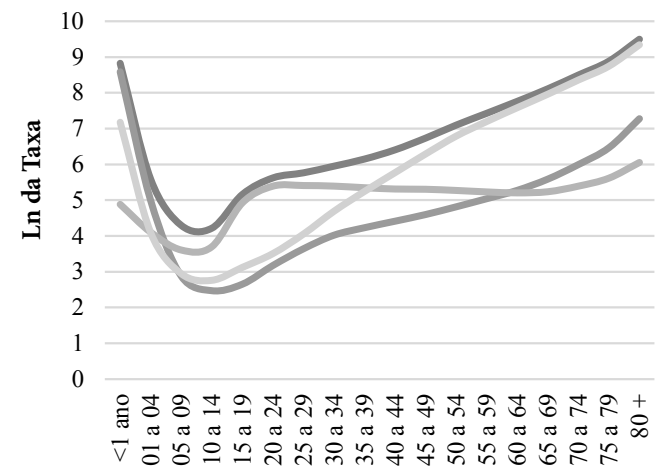

Grupo Etário

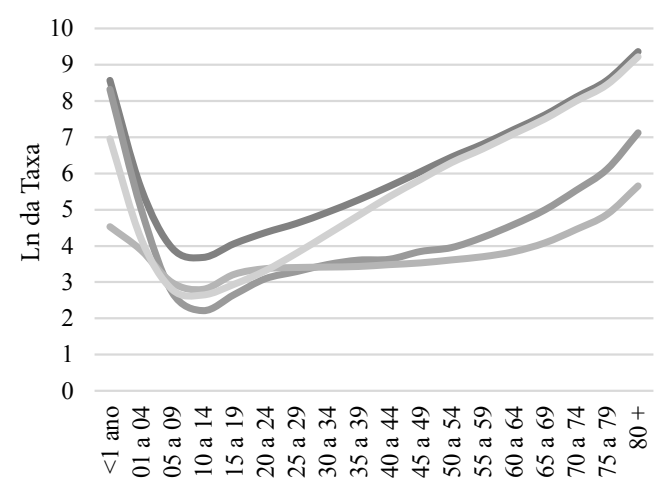

Grupo Etário

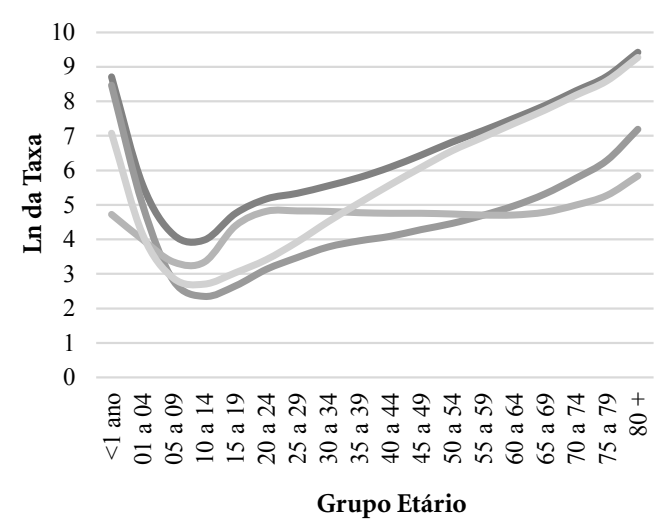

Mortalidade

1990

Figura 4. Curvas de Mortalidade e DALY por grupos de causa segundo sexo. Brasil, 1990-2019.

tre a expectativa de vida e a expectativa de vida saudável demonstrou que não necessariamente os anos prolongados de vida significaram tempo de vida saudável ${ }^{19}$.

Posteriormente, Souza et al..$^{20}$ identificaram, com dados até 2015, que o país possui uma re- dução importante da mortalidade por doenças transmissíveis e outras causas evitáveis, mas observaram aumento progressivo da mortalidade por doenças crônicas. A nossa análise avança neste diagnóstico, não apenas com relação ao tempo de observação, mas apresentando tan- 


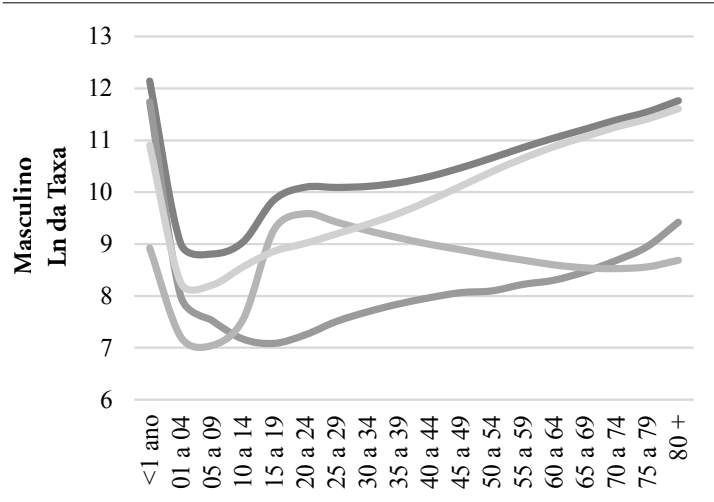

Grupo Etário
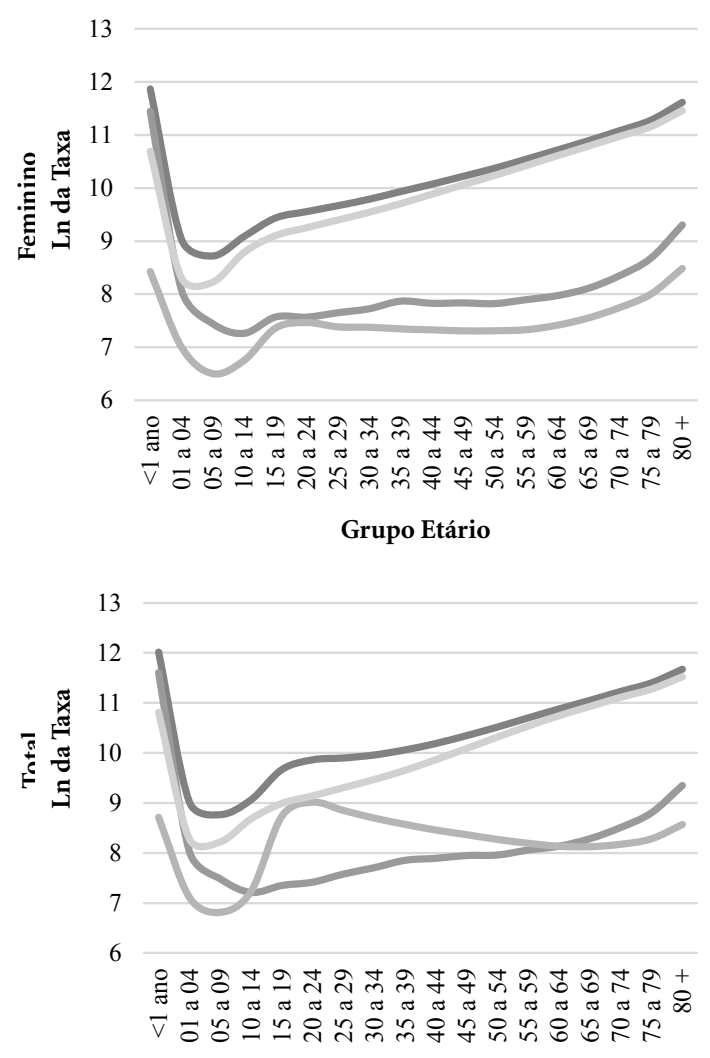

Grupo Etário

$$
\text { DALY }
$$
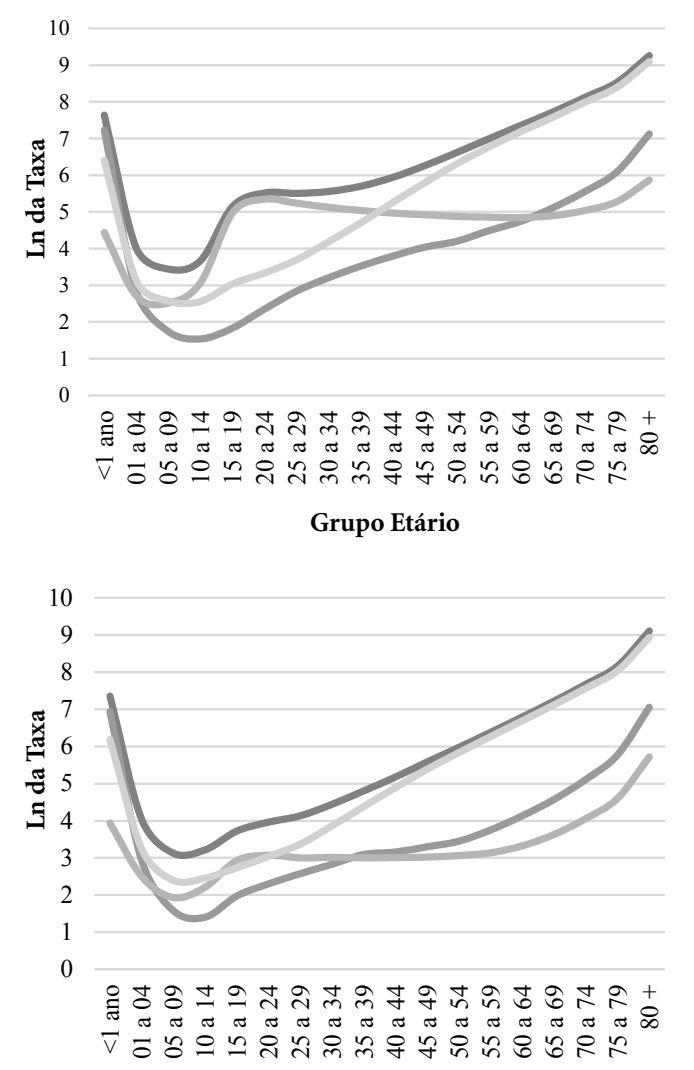

Grupo Etário

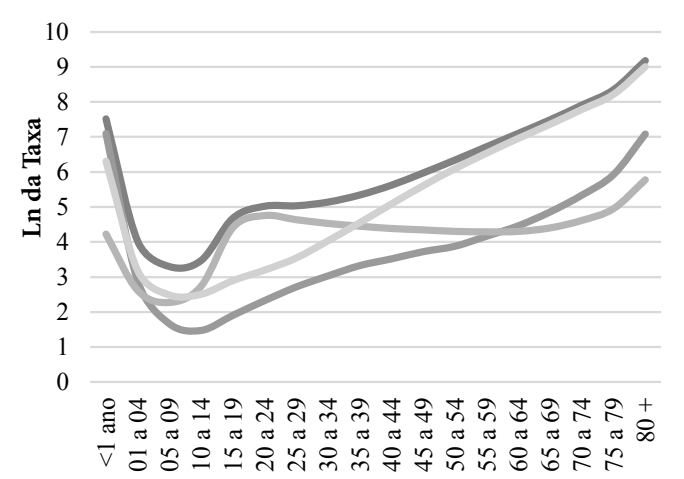

Grupo Etário

Mortalidade

2019

Legenda: Infecciosas $\longrightarrow$ DCNT $\longrightarrow$ Total

Figura 4. Curvas de Mortalidade e DALY por grupos de causa segundo sexo. Brasil, 1990-2019.

Fonte: GBD, 2021.

to o indicador das mortes quanto do indicador global de carga de doença (DALY). Além disso, apontamos o diferencial de sexo, e descrevemos o efeito da estrutura etária para explicar parcialmente a mudança no tempo. Estas características, eminentemente demográficas, são centrais para uma discussão adequada das mudanças no perfil de adoecimento e morte, e para a definição de populações alvo para as intervenções em saúde pública $^{21}$. 
Tabela 1. Tendência da taxa de mortalidade e DALY e taxa de incremento anual, segundo grupo de causa e de sexo. Brasil, 1990-2019.

\begin{tabular}{|c|c|c|c|c|c|c|}
\hline & $\boldsymbol{\beta}$ & EP & p valor & DW & $\mathbf{R}^{2}$ & $\begin{array}{c}\text { Taxa de } \\
\text { Incremento Anual } \\
\text { (IC 95\%) }\end{array}$ \\
\hline \multicolumn{7}{|c|}{ Doenças Infecciosas e Parasitárias } \\
\hline DALY feminino & $-34,76$ & 3,12 & $<0,001$ & 1,668 & 0,970 & $-1,64(-1,72 ;-1,55)$ \\
\hline DALY masculino & $-65,55$ & 5,96 & $<0,001$ & 1,435 & 0,984 & $-0,73(-0,80 ;-0,66)$ \\
\hline DALY total & $-49,27$ & 3,80 & $<0,001$ & 1,455 & 0,987 & $-0,90(-0,97 ;-0,83)$ \\
\hline Mortalidade Feminina & $-0,51$ & 0,06 & $<0,001$ & 1,583 & 0,963 & $-1,37(-1,50 ;-1,24)$ \\
\hline Mortalidade Masculina & $-1,24$ & 0,12 & $<0,001$ & 1,482 & 0,983 & $-0,79(-0,87 ;-0,71)$ \\
\hline Mortalidade Total & $-0,86$ & 0,08 & $<0,001$ & 1,548 & 0,982 & $-0,86(-0,88 ;-0,84)$ \\
\hline \multicolumn{7}{|c|}{ Doenças Crônicas Não Transmissíveis } \\
\hline DALY feminino & $-191,75$ & 3,25 & $<0,001$ & 1,916 & 0,998 & $-0,94(-0,98 ;-0,89)$ \\
\hline DALY masculino & $-251,11$ & 4,59 & $<0,001$ & 1,897 & 0,996 & $-1,01(-1,03 ;-0,98)$ \\
\hline DALY total & $-221,75$ & 3,60 & $<0,001$ & 1,933 & 0,997 & $-0,95(-0,96 ;-0,93)$ \\
\hline Mortalidade Feminina & $-8,44$ & 0,61 & $<0,001$ & 2,010 & 0,987 & $-1,43(-1,48 ;-1,38)$ \\
\hline Mortalidade Masculina & $-7,45$ & 0,68 & $<0,001$ & 1,853 & 0,976 & $-1,49(-1,56 ;-1,43)$ \\
\hline Mortalidade Total & $-9,21$ & 0,41 & $<0,001$ & 1,875 & 0,991 & $-1,32(-1,36 ;-1,28)$ \\
\hline \multicolumn{7}{|l|}{ Causas externas } \\
\hline DALY feminino & $-254,61$ & 21,29 & $<0,001$ & 1,696 & 0,861 & $-3,29(-3,39 ;-3,19)$ \\
\hline DALY masculino & $-323,24$ & 21,56 & $<0,001$ & 1,604 & 0,872 & $-3,50(-3,60 ;-3,41)$ \\
\hline DALY total & $-289,17$ & 23,14 & $<0,001$ & 1,631 & 0,868 & $-3,41(-3,51 ;-3,32)$ \\
\hline Mortalidade Feminina & $-3,33$ & 0,343 & $<0,001$ & 1,473 & 0,839 & $-2,77(-2,95 ;-2,60)$ \\
\hline Mortalidade Masculina & $-4,52$ & 0,470 & $<0,001$ & 1,519 & 0,859 & $-2,82(-3,00 ;-2,64)$ \\
\hline Mortalidade Total & $-3,91$ & 0,390 & $<0,001$ & 1,481 & 0,851 & $-2,81(-3,00 ;-2,65)$ \\
\hline \multicolumn{7}{|l|}{ Total } \\
\hline DALY feminino & $-482,98$ & 32,68 & $<0,001$ & 1,517 & 0,976 & $-1,47(-1,51 ;-1,43)$ \\
\hline DALY masculino & $-656,34$ & 46,99 & $<0,001$ & 1,492 & 0,978 & $-1,48(-1,52 ;-1,44)$ \\
\hline DALY total & $-569,70$ & 39,68 & $<0,001$ & 1,432 & 0,977 & $-1,48(-1,53 ;-1,44)$ \\
\hline Mortalidade Feminina & $-11,29$ & 1,11 & $<0,001$ & 1,493 & 0,953 & $-1,71(-1,79 ;-1,62)$ \\
\hline Mortalidade Masculina & $-15,26$ & 1,32 & $<0,001$ & 1,842 & 0,978 & $-1,47(-1,54 ;-1,41)$ \\
\hline Mortalidade Total & $-13,31$ & 1,25 & $<0,001$ & 1,680 & 0,966 & $-1,59(-1,67 ;-1,52)$ \\
\hline
\end{tabular}

Fonte: GBD, 2021.

O Brasil tem demonstrado um notável resultado na diminuição da mortalidade precoce. A proporção de óbitos ocorridos antes dos 20 anos de idade mudou de $12,2 \%$ para $7,4 \%$ entre 2000-2010. No mesmo período, a probabilidade de óbito no primeiro ano de vida teve uma queda de 26,6 para 16,2 por mil nascidos vivos ${ }^{22}$. Em 2010, as DCNT corresponderam a 73,9\% dos óbitos no Brasil, sendo que $80,1 \%$ foram causados por diabetes, câncer, DPOC e doenças cardiovasculares $^{23}$. Há, contudo, no Brasil, uma acumulação epidemiológica com a coexistência de elevada morbidade e mortalidade por doenças crônico-degenerativas, bem como altas incidência e prevalência, também, de doenças infecciosas e parasitárias, que apresentam alta mortalidade em comparação a países desenvolvidos ${ }^{22}$.
Ainda, verifica-se que há uma polarização geográfica, indicando a existência de regiões com distintos padrões de saúde, com algumas delas se assemelhando aos países desenvolvidos, e outras mais próximas dos países em desenvolvimento. Esta segregação espacial é resultado de uma polarização social que é marcada pela distinção na magnitude dos indicadores de morbimortalidade entre grupos populacionais, ainda que pertencentes a uma mesma região, estado ou cidade. Trata-se, pois, de uma manifestação das desigualdades de renda e de acesso a bens essenciais, como moradia, educação, saneamento e acesso aos serviços de saúde. Desta forma, ainda que haja certa convergência nos indicadores regionais de mortalidade, o Brasil segue apresentando certas peculiaridades na transição ${ }^{11}$. 
Mediante este cenário brasileiro foi desenvolvido no âmbito do Sistema Único de Saúde (SUS) o Plano de Ações Estratégicas para o Enfrentamento das Doenças Crônicas não Transmissíveis (DCNT), e, a partir de então, realizouse pesquisas nacionais e monitoramento das metas de redução de mortalidade e fatores de risco nos últimos anos. O que se observou foi um avanço referente à vigilância, promoção da saúde e cuidado integral. De modo geral, as metas do Plano vêm sendo alcançadas ${ }^{24}$. De fato, houve declínio médio de $2,5 \%$ ao ano no conjunto das quatro principais DCNT no Brasil entre $2000 \mathrm{e}$ 2013, em todas as regiões e unidades federativas. A probabilidade de morte foi reduzida de $30 \%$, em 2000, para 26,1\% em 2013, e estima-se que caia para $20,5 \%$ em $2025^{25}$. Com isso, há uma expectativa de que o Brasil consiga atingir a meta global prevista de redução de $25 \%$.

Em relação às doenças infecciosas e parasitárias, houve redução da sua contribuição relativa, tanto na mortalidade quanto no DALY, sobretudo nas faixas etárias de crianças menores que 10 anos e, principalmente, entre as menores de 1 ano. No entanto, é importante perceber que, mesmo que o padrão da mortalidade seja a redução quase linear das doenças infecciosas e das causas associadas ao período perinatal, estes grupos ainda têm importante carga de doenças, possivelmente por ocorrerem em grupos de crianças e pré-adolescentes, de forma que, apesar da menor expressão desta faixa etária, o cômputo dos anos com algum tipo de consequência destas doenças persiste por quase toda a vida ${ }^{26}$.

O grupo de causas que manteve peso semelhante para a mortalidade e para a morbidade, no período estudado, foi o das causas externas. Por esta razão, considera-se que, no Brasil, assim como na América Latina, em geral, é importante destacar a chamada tripla carga de doenças, destacando as causas externas dos demais agravos não transmissíveis, tamanha sua importância no cenário epidemiológico ${ }^{27,28}$. Os avanços tímidos ou inexistentes na superação das mortes por causas externas no país preocupam por refletirem a desigualdade socioeconômica e regional do país. Domicílios de famílias menos abastadas e localizados em áreas com menor acesso aos serviços básicos de infraestrutura urbana apresentam maiores chances de observarem óbitos de jovens adultos $^{29}$. Além disso, as Regiões Norte e Nordeste observaram entre 2000 e 2010 avanços mais tímidos em seus níveis de expectativa de vida, sobretudo, por conta da contribuição da mortalidade de homens entre 15-29 anos ${ }^{11}$.
As limitações e exceções ao modelo original da transição epidemiológica proposto por Omran ${ }^{2}$, estimularam o surgimento de propostas alternativas à teoria clássica, como a transição para a saúde ${ }^{4,30-32}$. Com esses termos, reconhece-se um modelo muito mais complexo do que a simples evolução da dinâmica dos perfis epidemiológicos. Frenk et al. ${ }^{33,34}$ avançaram em uma proposta teórica que busca complementar os diferentes modelos de transição apresentados acima e gerar um quadro analítico adaptável à maneira como se produziu o declínio da mortalidade na América Latina, seus determinantes e evolução dos padrões de morbidade e mortalidade existentes. Os autores mantêm a proposta analítica de $\mathrm{Omran}^{2}$, e acrescentaram três principais mudanças no perfil epidemiológico: 1) a substituição das causas de mortes por doenças infecciosas, doenças não transmissíveis e causas externas; 2) o deslocamento da maior carga de morbidade e mortalidade de jovens para idosos; 3) predominância de morbidade sobre mortalidade. Este modelo, caracterizado principalmente por uma sobreposição de estágios, refere-se à incidência simultânea de doenças infecciosas e doenças crônicas, com o reconhecimento da heterogeneidade presente internamente nos países, e parece mais adequado para a explicação da sobreposição de estágios observada em locais com relativa desigualdade social e geográfica, como é o caso do Brasil.

Diante de um quadro de acelerado processo de envelhecimento populacional, a perspectiva é de que as tendências no perfil epidemiológico brasileiro trarão ao Sistema Único de Saúde o desafio permanente de revisão do modelo de cuidados de atenção à saúde ${ }^{35,36}$. Ainda que não mude em sua essência, a transição de morbimortalidade requer um esforço contínuo de monitoramento e avaliação da eficácia e da efetividade das medidas de saúde pública ${ }^{37,38}$. Para isso, é essencial dar maior protagonismo às ações de vigilância em saúde ${ }^{39}$. Destacamos a necessidade de qualificação de técnicos para as áreas de epidemiologia de campo, análise de situação de saúde e métodos quantitativos, para que os indicadores possam ser adequadamente construídos, criticamente analisados e utilizados no processo de tomada de decisão no âmbito do SUS.

\section{Considerações finais}

Os dados do Estudo Carga Global de Doenças (GBD) disponíveis para o Brasil, permitem traçar 
um rico panorama da situação de saúde da população brasileira ao longo dos últimos 30 anos, período sob a égide do Sistema Único de Saúde (SUS). Nota-se, de forma inequívoca, que o país se encontra em avançado estágio nos seus processos de transição demográfica e epidemiológica, exibindo ganhos expressivos na expectativa média de vida de sua população, ao mesmo tempo em que diminuiu progressivamente o peso da mortalidade por doenças infecto-parasitárias, de uma forma geral, e aumentou o peso da morbimortalidade por doenças crônicas não transmissíveis (DCNT), especialmente nos grupos etários mais idosos. Tais transformações constituem forte evidência das conquistas e da importância desempenhada pelo SUS na promoção da saúde da população brasileira, ainda que existam diversos desafios a serem superados.

Manifestando-se sob a forma de uma polarização epidemiológica multidirecional, é possível afirmar que a transição epidemiológica brasileira continua ainda caracterizada por uma tripla carga de doenças, em que altas taxas de morbimortalidade por doenças crônicas não transmissíveis, especialmente na população idosa (característi- cas de regiões em avançado estágio de transição da saúde), coexistem com uma elevada incidência e prevalência de doenças infecto-parasitárias, especialmente na população abaixo dos 10 anos, e de causas externas, com destaque para os homicídios, na população masculina jovem (15-29 anos). Tais peculiaridades da transição epidemiológica brasileira são um sério desafio à melhoria das condições de vida e de saúde da população do país e precisam ser levadas em consideração no desenho de políticas públicas de saúde.

Para traçar estratégias para o enfrentamento de tais desafios é necessário conhecer o padrão de estado de saúde e suas tendências nas últimas décadas, dentre eles, as novas epidemias, as doenças negligenciadas, o controle dos vetores, a magnitude das doenças crônicas não transmissíveis e da violência. Além disso, é preciso reconhecer, também, as enormes desigualdades regionais e os distintos contextos socioculturais em que se dão os processos de transição demográfica e epidemiológica no Brasil. Tais processos mostram-se intimamente ligados aos padrões socioespaciais de distribuição da riqueza e dos serviços de saúde e de saneamento básico no território nacional.

\section{Colaboradores}

Todos os autores contribuíram com a concepção do estudo, coleta e análise de dados, redação e revisão crítica do manuscrito.

\section{Referências}

1. United Nations, Department of Economic and Social Affairs, Population Division. World Population Prospects 2019: Data Booklet (ST/ESA/SER.A/424). [cited 2020 Nov 10]. Available from: https://population.un.org/wpp/Publications/Files/WPP2019_DataBooklet.pdf. Acesso em novembro de 2020

2. Omran AR. The epidemiologic transition: a theory of the epidemiology of population change. Milbank Mem Fund Q 1971; 49:509-538.

3. Mesle F, Vallin J. The health transition: trends and prospects. Demography, analysis and synthesis. A treatise in demography. New York: Elsevier; 2006. p. 247-602.

4. Caselli G, Meslé F, Vallin J. Epidemiologic transition theory exceptions. Genus 2002; 58:9-52.

5. Omran AR. The epidemiologic transition theory. A preliminary update. J Trop Pediatr 1983; 29:305-316.

6. Omran AR. The epidemiologic transition theory revisited thirty years later. World Health Stat Q 1998; 51:99-119.

7. Vallin J. Diseases, deaths, and life expectancy. Genus 2005; 61:279-296.

8. Olshansky S, Ault B. The fourth stage of epidemiology transition: The age of delayed degenerative diseases. Milbank Memorial Fund Quarterly 1986; 64(3):355391. 
9. Horiuchi H. Epidemiological transitions in human history. Proceedings of the Symposium on health and mortality: Issues of Global Concern, Brussels; 1997 Nov 19-22; New York: United Nations; 1997.

10. Def BK. Beyond the 'transition' frameworks: the cross-continuum of health, disease and mortality framework. Glob Health Action 2014; 7(10):3402.

11. Borges GM. A transição da saúde no Brasil: variações regionais e divergência/convergência na mortalidade. Cad Saude Publica 2017; 33(8):e00080316.

12. Paim JS. Sistema Único de Saúde (SUS) aos 30 anos. Cien Saude Colet 2018; 23(6):1723-1728.

13. Malta DC, Felisbino-Mendes MS, Machado ÍE, Passos VMA, Abreu DMX, Ishitani LH, Velásquez-Meléndez G, Carneiro M, Mooney M, Naghavi M. Fatores de risco relacionados à carga global de doença do Brasil e Unidades Federadas, 2015. Rev Bras Epidemiol 2017; 20(1):217-232.

14. GBD 2017 Risk Factor Collaborators. Global, regional, and national comparative risk assessment of 84 behavioural, environmental and occupational, and metabolic risks or clusters of risks for 195 countries and territories, 1990-2017: a systematic analysis for the Global Burden of Disease Study 2017. Lancet 2018; 392(10159):1923-1994.

15. Gibney K, Sinclair M, O’Toole J, Leder K. Using disability-adjusted life years to set health-based targets: a novel use of an established burden of disease metric. $J$ Public Health Policy 2013; 34(3):439-446.

16. Doll R, Payne P, Waterhouse J, editors. Cancer incidence in five continents: a technical report. Berlin: Springer-Verlag; 1966.

17. Antunes JLF, Cardoso MRA. Uso da análise de séries temporais em estudos epidemiológicos. Epidemiol Serv Saude 2015; 24(3):565-576.

18. Marinho F, Passos VMA, França EB. Novo século, novos desafios: mudança no perfil da carga de doença no Brasil de 1990 a 2010. Epidemiol Serv Saude 2016; 25(4):713-724.

19. Salomon JA, Wang H, Freeman MK, Vos T, Flaxman $\mathrm{AD}$, Lopez AD, Murray CJ. Healthy life expectancy for 187 countries, 1990-2010: a systematic analysis for the Global Burden Disease Study 2010. Lancet 2012; 380:2144-2162.

20. Pinto LF, Freitas MPS, Sant'Anna AW. Sistemas Nacionais de Informação e levantamentos populacionais: algumas contribuições do Ministério da Saúde e do IBGE para a análise das capitais brasileiras nos últimos 30 anos. Cien Saude Colet 2018; 23(6):1859-1870.

21. Souza MFM, Malta DC, Franca EBB, Lima M. Transição da saúde e da doença no Brasil e nas Unidades Federadas durante os 30 anos do Sistema Único de Saúde. Cien Saude Coletiva 2018; 23(6):1737-1750.

22. Araújo JD. Polarização epidemiológica no Brasil. Epidemiol Serv Saude 2012; 21(4):533-538.

23. Campolina AG, Adami F, Santos JL, Lebrão ML. A transição de saúde e as mudanças na expectativa de vida saudável da população idosa: possíveis impactos da prevenção de doenças crônicas. Cad Saude Publica 2013; 29(6):1217-1229.

24. Malta DC, Oliveits TP, Santos MAS, Andrade SSCA, Silva MMA. Avanços do Plano de Ações Estratégicas para o Enfrentamento das Doenças Crônicas não Transmissíveis no Brasil, 2011-2015. Epidemiol Serv Saude 2016; 25(2):373-390.
25. Malta DC, Andrade SSCA, Oliveira TP, Moura L, Prado RR, Souza MFM. Probabilidade de morte prematura por doenças crônicas não transmissíveis, Brasil e regiões, projeções para 2025. Rev Brasil Epidemio 2019; 22:e190030.

26. Colzani E. Beyond morbidity and mortality: the burden of infectious diseases on healthcare services. Epidemiol Infect 2019; 147:e251.

27. Peixoto SV. The triple burden of health problems and the challenges for the Unified Health System. Cien Saude Coletiva 2020; 25(8):2913.

28. Alvarez JA, Aburto JM, Canudas-Romo V. Latin American convergence and divergence towards the mortality profiles of developed countries. Popul Stud (Camb). 2020; 74(1):75-92.

29. Pereira F, Queiroz BL. Differences in mortality in Brazilian youth: the importance of household socioeconomic factors and living conditions in Brazilian municipalities and states. Cad Saude Publica 2016; 32(9):e00109315.

30. Vallin J, Meslé F. Convergences and divergences in mortality. A new approach to health transition. Demographic Research 2004; 2(2):11-14.

31. Caldwell J. Health Transition: the cultural, social and behavioral determinants of health in the third World. Soc Sci Med 1993; 36(2):125-135.

32. Cleland J, Hill A. The health transition: methods and measures. Canberra: Australian National University; 1991.

33. Frenk J, Bobadilla JL, Stern C, Frejka T, Lozano R. Elements for a theory of the health transition. Health Transit Rev 1991; 1:21-38.

34. Frenk J, Bobadilla JL, Sepúlveda J, Cervantes ML. Health transition in middle-income countries: new challenges for health care. Health Policy Plan 1989; 4:29-39.

35. Marinho F, Passos VMA, França EB. Novo século, novos desafios: mudança no perfil da carga de doença no Brasil de 1990 a 2010. Epidemiol Serv Saude 2016; 25(4):713-724.

36. Noronha JC, Noronha GS; Pereira TR, Costa AM. Notas sobre o futuro do SUS: breve exame de caminhos e descaminhos trilhados em um horizonte de incertezas e desalentos. Cien Saude Coletiva 2018; 23(6):20512059.

37. Schramm JMA, Oliveira AF, Leite IC, Valente JG, Gadelha AMJ, Portela MC, Campos MR. Transição epidemiológica e o estudo de carga de doença no Brasil. Cien Saude Coletiva 2004; 9(4):897-908.

38. Souza MFM, Malta DC, França EB, Barreto ML. Transição da saúde e da doença no Brasil e nas Unidades Federadas durante os 30 anos do Sistema Único de Saúde. Cien Saude Colet 2018; 23(6):1737-1750.

39. Teixeira MG, Carmo EH, Oliveira WK, Penna GO. Vigilância em Saúde no SUS - construção, efeitos e perspectivas. Cien Saude Colet 2018; 23(6):1811-1818.

Artigo apresentado em 20/11/2020

Aprovado em 20/05/2021

Versão final apresentada em 22/05/2021

Editores-chefes: Romeu Gomes, Antônio Augusto Moura da Silva 